Bundesgesundheitsbl 2017 $60: 410-418$ DOI 10.1007/s00103-017-2521-1

Online publiziert: 3. März 2017

(c) Der/die Autor(en) 2017. Dieser Artikel ist eine Open-Access-Publikation.

CrossMark

Ilona M. Punt ${ }^{1} \cdot$ Roel van der Most ${ }^{2} \cdot$ Bart C. Bongers ${ }^{1} \cdot$ Anouk Didden $^{3}$. Erik H. J. Hulzebos ${ }^{4}$. Jaap J. Dronkers ${ }^{5} \cdot$ Nico L. U. van Meeteren ${ }^{1,6}$

'Institut für Epidemiologie, Maastricht University, CAPHRI, Maastricht, Niederlande

${ }^{2}$ Chirurgisch Traumatologisches Zentrum, Fachbereich Orthopädie, Asklepios Klinik St. Georg, Hamburg, Deutschland

${ }^{3}$ Abt. für Physiotherapie, Maastricht University Medical Center, Maastricht, Niederlande

${ }^{4}$ University Children's Hospital and Medical Centre Utrecht, Child and Development and Exercise Centre, University Medical Centre Utrecht, Utrecht, Niederlande

${ }^{5}$ Abt. für Physiotherapie, Gelderse Vallei Hospital, Ede, Niederlande

${ }^{6}$ Topsector Life Sciences and Health (Health Holland), Den Haag, Niederlande

\title{
Verbesserung des prä- und postoperativen Behandlungskonzepts
}

\section{Große elektiv-chirurgische Eingriffe}

In den letzten Jahrzehnten haben Fortschritte in der Chirurgie und Anästhesiologie zu verbesserten Behandlungskonzepten und postoperativen Ergebnissen geführt. Allerdings sind chirurgische Eingriffe mit möglichen negativen $\mathrm{Ne}$ benwirkungen wie perioperativen Komplikationen, verlängerter Liegedauer sowie einer verzögerten Integration des $\mathrm{Pa}$ tienten im alltäglichen Leben behaftet. Vor allem bei älteren und fragilen $\mathrm{Pa}$ tienten wird der körperliche Abbau vor (in den Wartephasen), während und nach dem Krankenhausaufenthalt als größter negativer Effekt gesehen [1].

Covinsky et al. [2] zeigten beispielsweise, dass bei $43 \%$ der auf eine Operation wartenden Patienten Zeichen des körperlichen Abbaus zu verzeichnen waren. Bei $34 \%$ der entlassenen Patienten wurden schlechtere funktionelle Scores erhoben [2]. Durch Kräfteverlust der unteren Extremitäten, Reduktion der Ausdauer- und kardiopulmonalen Funktionsfähigkeit führt die postoperative Bettruhe $\mathrm{zu}$ einem zunehmenden Verlust der körperlichen Funktionsfähigkeit, was in der angelsächsischen Literatur auch als „hospitalization associated disability“ bezeichnet wird [3]. Es wird angenommen, dass dieses Phänomen iatrogen bedingt ist, dem vorgebeugt werden kann [4]. Damit gefährdete Patienten nicht unterhalb der kritischen Schwelle (die körperliche Funktionsfähigkeit ist zu niedrig, um sich ohne Komplikationen von einem chirurgischen Eingriff zu erholen) geraten, ist ein präoperatives Screeningprogramm unentbehrlich. Patienten die im Screeningprogramm mit einem Hochrisikoprofil gekennzeichnet werden, sollten demnach an einer präoperativen, funktionsfähigkeitssteigernden Trainingstherapie (Prähabilitation) teilnehmen [5].

\section{Prähabilitation}

Prähabilitation wird als ein Prozess definiert, der aus Vorbereitungs- und Behandlungskontinuität besteht und bei dem ein Hochrisikopatient von der Indikationsstellung, durch die stationäre Phase bis zum chirurgischen Eingriff begleitet wird. Das Ziel ist es, proaktiv die körperliche Funktionsfähigkeit $\mathrm{zu}$ steigern und die physiologischen Reserven anzuheben. Prähabilitationspatienten haben dadurch eine größere Chance, trotz negativer Begleiterscheinungen eines größeren chirurgischen Eingriffs schneller zu genesen. Die kritische Leistungszone wird somit nicht erreicht, wodurch eine Reduktion der Komplikationen sowie bessere postoperativen Ergebnisse erzielt werden kön- nen. Zu Beginn der Prähabilitation wird von jedem Patienten ein persönliches Risikoprofil erstellt und die minimale Leistungsgrenze berechnet. Dadurch wird dem behandelnden Arzt ermöglicht, je nach Zustand des Patienten einen individualisierten Therapieplan festzulegen, der die allgemeine Gesundheit und die gesundheitsbezogene Leistungsfähigkeit steigert. Die Prähabilitation ermöglicht Patienten und Therapeuten, proaktiv und gezielt die höchstmögliche Leistungsfähigkeit zu erreichen und somit Morbidität, Mortalität, Verweildauer und die Zahl erneuter stationärer Aufnahmen zu reduzieren (• Abb. 1). Zusammengefasst wandelt die Prähabilitation die inaktive Wartephase in eine (pro)aktive Stärkungsphase um.

Die Prähabilitation steigert die pulmonale, kardiovaskuläre und muskuläre Leistungsfähigkeit, wodurch die körperliche Funktionsfähigkeit des Patienten verbessert wird, damit das Anpassungsvermögen des Körpers auf die perioperative Stressreaktion vorbereitet ist [6]. Außerdem bietet sie einen proaktiven Kontext, indem aufeinanderfolgende Prozesse eine lückenlose Versorgung bei der Behandlung von elektiven Fällen ermöglichen (• Abb. 2). Die Prähabilitation hält sowohl Niedrig- als auch Hochrisikopatienten perioperativ und nach der 


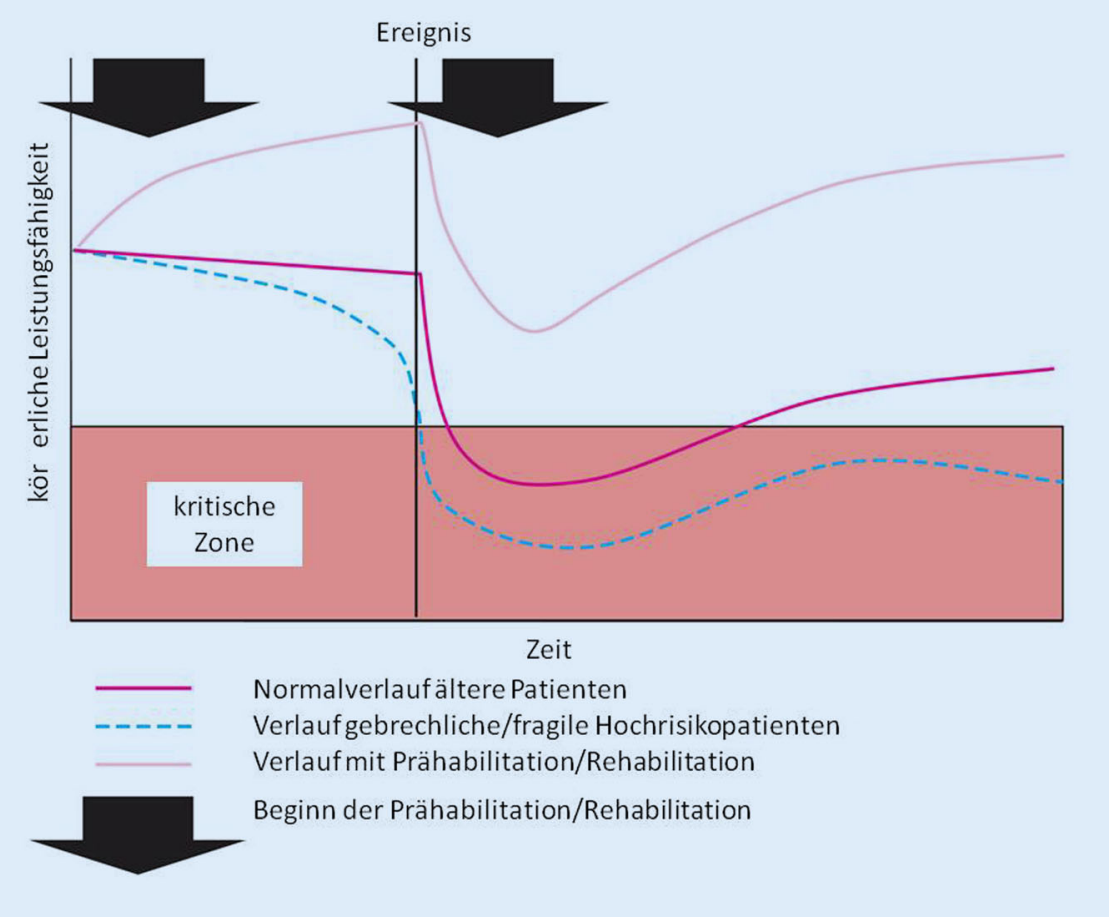

Abb. $1 \Delta$ Ältere Patienten durchlaufen während des stationären Aufenthalts für eine Elektivoperation eine typische Leistungskurve. Die körperliche Leistung fällt durch den Einfluss der Operation zuerst ab, um postoperativ wieder anzusteigen. Die meisten Patienten, die über eine adäquate Stressreaktion verfügen, durchlaufen eine normale Kurve und haben bei der Entlassung wieder ihr präoperatives Niveau erreicht (mittlere Linie). Bei Hochrisikopatienten (untere gestrichelte Linie) kann die Wartephase die Reduktion der Leistungsfähigkeit in die kritische Zone verstärken. Die Erholungsphase nach der Operation wird somit verlängert. Am Tag der geplanten Entlassung könnte der Anfangswert der körperlichen Leistung noch nicht erreicht sein. Die Prähabilitation kann die körperliche präoperative Leistungsfähigkeit von Hochrisikopatienten (obere Linie) anheben, wodurch einem physiologischen Rückfall bis in die kritische Zone vorgebeugt werden kann [1]. Der linke Pfeil gibt den Beginn der Prähabilitation an. Diese fängt direkt nach dem präoperativen Risikoassessment an. Der zweite Pfeil markiert den Anfang der klassischen postoperativen Physiotherapie/Rehabilitation

Entlassung auf einem höchstmöglichen Aktivitätsniveau [1].

Die wichtigsten Aspekte des ambulanten Trainings (zu Hause) bei Hochrisikopatienten sind [7]:

- Die Kurz- und Langzeitziele sollten mit dem Patienten im Voraus besprochen werden (gemeinsame Trainingsziele). Das Training sollte auf die Verbesserung der Aktivitäten des täglichen Lebens (ADL) fokussiert sein $[8,9]$.

- Da die körperliche Leistungsfähigkeit stark mit der Kraft der unteren Extremitäten korreliert, sollten diese Muskelgruppen auch zur Steigerung der ADL-Funktion im Fokus der Behandlung stehen. Außerdem wird der Patient durch Training des kardiovaskulären Systems auf die Zunahme des metabolischen Bedarfs nach der Operation vorbereitet. Die Zunahme des metabolischen Bedarfs ist bedingt durch Stressreaktionen (hormonelle Veränderungen und Wundheilungsprozesse, Blutverlust), die durch einen operativen Eingriff hervorgerufen werden [10].

- Bei den patientenspezifischen Übungen sollten nicht nur die Intensität und die Zahl der Übungen im Verlauf gesteigert werden, sondern auch die Komplexität und Variabilität $[11,12]$.

- Aufgrund der relativ kurzen Zeitspanne zwischen Indikation und Operation ist ein intensives Trainingsprogramm notwendig [13]. Das Ziel ist, durch überschwellige Reize eine biopositive Anpassung zu erzeugen und somit die kardiopulmonale und muskuläre bzw. sensomotorische Leistungsfähigkeit in dieser kurzen
Zeit zu steigern, ohne den Patienten in die Erschöpfung zu führen. Vor dem Beginn eines intensiven Trainings sollte der Behandler eine Risikountersuchung zur Abschätzung trainingsassoziierter Komplikationen durchführen. Die persönliche Erfahrung des Therapeuten sowie die Erhebung des Risikoassessments mit Fragebögen, beispielsweise das Physical Activity Readiness Questionnaire (PAR-Q), spielen hier eine Rolle. Gegebenenfalls wird die Einschätzung eines Spezialisten eingeholt (z. B. eines Kardiologen).

- Die Planung, der Inhalt, die Struktur, die Durchführung und die Überwachung der Trainingstherapie sollten in enger Zusammenarbeit mit dem Hochrisikopatienten und in seiner eigenen häuslichen Umgebung stattfinden. Ältere fragilere Patienten neigen eher dazu, an „homebased“ als an „clinic-based“ Therapien teilzunehmen [14-16]. Bei diesen Patienten könnte schließlich die Anreise zur Klinik/Praxis erhebliche Probleme (Reisedistanz, Treppe, Hilfsmittel) verursachen und dafür sorgen, dass bei der Ankunft nicht mehr ausreichend Energie für die Übungstherapie übrig bleibt [14]. Das Ziel der Übungstherapie ist u. a. praktische Lösungen anzubieten, die von der Wohnsituation des Einzelnen und körperlichen Einschränkungen des Patienten abhängig sind. Beim Üben in häuslicher Umgebung werden patientenspezifische Probleme schneller entdeckt und Lösungen einfacher gefunden.

- Das Training sollte sowohl durch den Patienten als auch durch den Physiotherapeuten mithilfe von Funktionstests überwacht werden, damit die Dosis der Therapieeinheiten engmaschig kontrolliert und angepasst werden kann $[17,18]$. Patienten sollten die Führung bei der Einschätzung des Therapieerfolgs und gegebenenfalls selbst die Initiative zur Übungssteigerung übernehmen, was eine höhere Motivation, eine Einhaltung des Therapieplans und Zufriedenheit zur Folge hat. 
Bundesgesundheitsbl 2017·60:410-418 DOI 10.1007/s00103-017-2521-1

(c) Der/die Autor(en) 2017. Dieser Artikel ist eine Open-Access-Publikation.

\section{M. Punt · R. van der Most • B. C. Bongers · A. Didden · E. H. J. Hulzebos · J. J. Dronkers · N. L. U. van Meeteren}

\section{Verbesserung des prä- und postoperativen Behandlungskonzepts. Große elektiv-chirurgische Eingriffe}

\section{Zusammenfassung}

Das Ziel der Chirurgie ist es, die Gesundheit des Patienten zu steigern. Allerdings sind chirurgische Eingriffe mit möglichen negativen Folgen wie perioperative Komplikationen und verlängerte Liegedauer behaftet. Außerdem kann das Erreichen der präoperativen körperlichen Funktionsfähigkeit verzögert werden. Speziell bei fragilen Patienten gefährden vor allem die Wartezeit bis zur Operation sowie der stationäre Aufenthalt ihre körperliche Verfassung.

Ziel des Better-in-better-out ${ }^{\mathrm{TM}}\left(\mathrm{BiBo}^{\mathrm{TM}}\right)$ Konzepts ist es, das Risiko für einen komplizierten postoperativen Verlauf durch Optimierung und Professionalisierung der perioperativen Behandlungsstrategien auf Basis physiotherapeutischer Aktivierungsmaßnahmen zu reduzieren. $\mathrm{BiBo}^{\mathrm{TM}}$ beinhaltet für jeden Patienten mit einem indizierten chirurgischen Elektiveingriff einen maßgeschneiderten 4-Punkte-Plan: 1) präoperative Risikoeinschätzung, 2) präoperative Patientenschulung, 3) präoperative Trainingstherapie für Hochrisikopatienten (Prähabilitation), 4) Postoperative Mobilisation und funktionelle Übungstherapie.

Das Ziel des präoperativen Screenings ist es, die fragilen Hochrisikopatienten frühzeitig zu identifizieren, damit diese rechtzeitig an einer ambulanten Übungstherapie (Prähabilitation) teilnehmen können. Durch eine gesteigerte körperliche Verfassung ist der Patient in der
Lage, den Auswirkungen großer chirurgischer Eingriffe besser standzuhalten, wodurch zum einen Nebenwirkungen reduziert und zum anderen bessere postoperative Ergebnisse erzielt werden. Neben der Prähabilitation sollte sich die Behandlungskultur und -infrastruktur grundsätzlich dahingehend ändern, dass Patienten auch nach der Entlassung körperlich, sozial und mental aktiv bleiben.

\section{Schlüsselwörter}

Hochrisikopatienten · Körperliche Aktivität . Perioperatives Risiko · Prähabilitation . Elektivchirurgie

\section{Improving pre- and perioperative hospital care. Major elective surgery}

\section{Abstract}

Surgery is aimed at improving a patient's health. However, surgery is plagued with a risk of negative consequences, such as perioperative complications and prolonged hospitalization. Also, achieving preoperative levels of physical functionality may be delayed. Above all, the "waiting" period before the operation and the period of hospitalisation endanger the state of health, especially in frail patients.

The Better in Better out ${ }^{\mathrm{TM}}\left(\mathrm{BiBo}^{\mathrm{TM}}\right)$ strategy is aimed at reducing the risk of a complicated postoperative course through the optimisation and professionalisation of perioperative treatment strategies in a physiotherapy activating context. $\mathrm{BiBo}^{\mathrm{Tm}}$ includes four steps towards optimising personalised health care in patients scheduled for elective surgery: 1) preoperative risk assessment, 2) preoperative patient education, 3) preoperative exercise therapy for high-risk patients (prehabilitation) and 4) postoperative mobilisation and functional exercise therapy.

Preoperative screening is aimed at identifying frail, high-risk patients at an early stage, and advising these high-risk patients to participate in outpatient exercise training (prehabilitation) as soon as possible. By improving preoperative physical fitness, a patient is able to better withstand the impact of major surgery and this will lead to both a reduced risk of negative side effects and better short-term outcomes as a result. Besides prehabilitation, treatment culture and infrastructure should be inherently changing in such a way that patients stay as active as they can, socially, mentally and physically after discharge.

Keywords

High-risk patients · Physical fitness · Risk stratification · Prehabilitation · Elective surgery

\section{Better-in-Better-out ${ }^{\mathrm{TM}}$-Strategie}

Die Better-in-Better-out ${ }^{\mathrm{Tx}}\left(\mathrm{BiBo}^{\mathrm{rm}}\right)$-Strategie wurde bereits 1996 entwickelt, um das perioperative Risiko eines komplizierten Krankheitsverlaufs nach chirurgischen Eingriffen zu reduzieren. Der Kern dieser Strategie ist eine Optimierung und Professionalisierung der vorstationären und perioperativen medizinischen Versorgung von Elektivpatienten durch aktives Anregen der körperlichen Leistungsfähigkeit.

Zusätzlich zum Konzept der Prähabilitation geht die $\mathrm{BiBo}^{\mathrm{Tw}}$-Strategie mit der präoperativen Risiko-Einschätzung einen Schritt weiter. $\mathrm{BiBo}^{\mathrm{TM}}$ beinhaltet die nachfolgenden 4 Schritte zur Optimierung der auf den Patienten zugeschnittenen Therapieangebote.

\section{Präoperativ}

\section{Risikoeinschätzung}

Die Patienten, bei denen ein größerer Elektiveingriff geplant ist, werden durch einen Physiotherapeuten im Krankenhaus untersucht und in Hoch- und Niedrigrisikogruppen eingeteilt [15, 19]. Diese präoperative Einschätzung basiert auf dem Aktivitäts- und Leistungsfähigkeitsniveau sowie auf persönlichen Fak- toren, der medizinischen Vorgeschichte (Alter, Body-Mass-Index [BMI], Diabetes, Herzfunktionsstörungen) und den kognitiven Fähigkeiten des Patienten. $\mathrm{Ob}$ und inwieweit das Vorhandensein von Risikofaktoren eine verzögerte $\mathrm{Ge}$ nesung vorhersagen kann, ist abhängig von der Art des Eingriffs und der Krankenhausversorgungsstruktur. Hauptsächlich wurden die kardiopulmonale Leistungsfähigkeit, die Muskelkraft und die Durchführung von körperlichen Aktivitäten als unabhängige Risikofaktoren für postoperative Komplikationen, das Erreichen der körperlichen Ausgangsfunktionsfähigkeit, Morbidität 


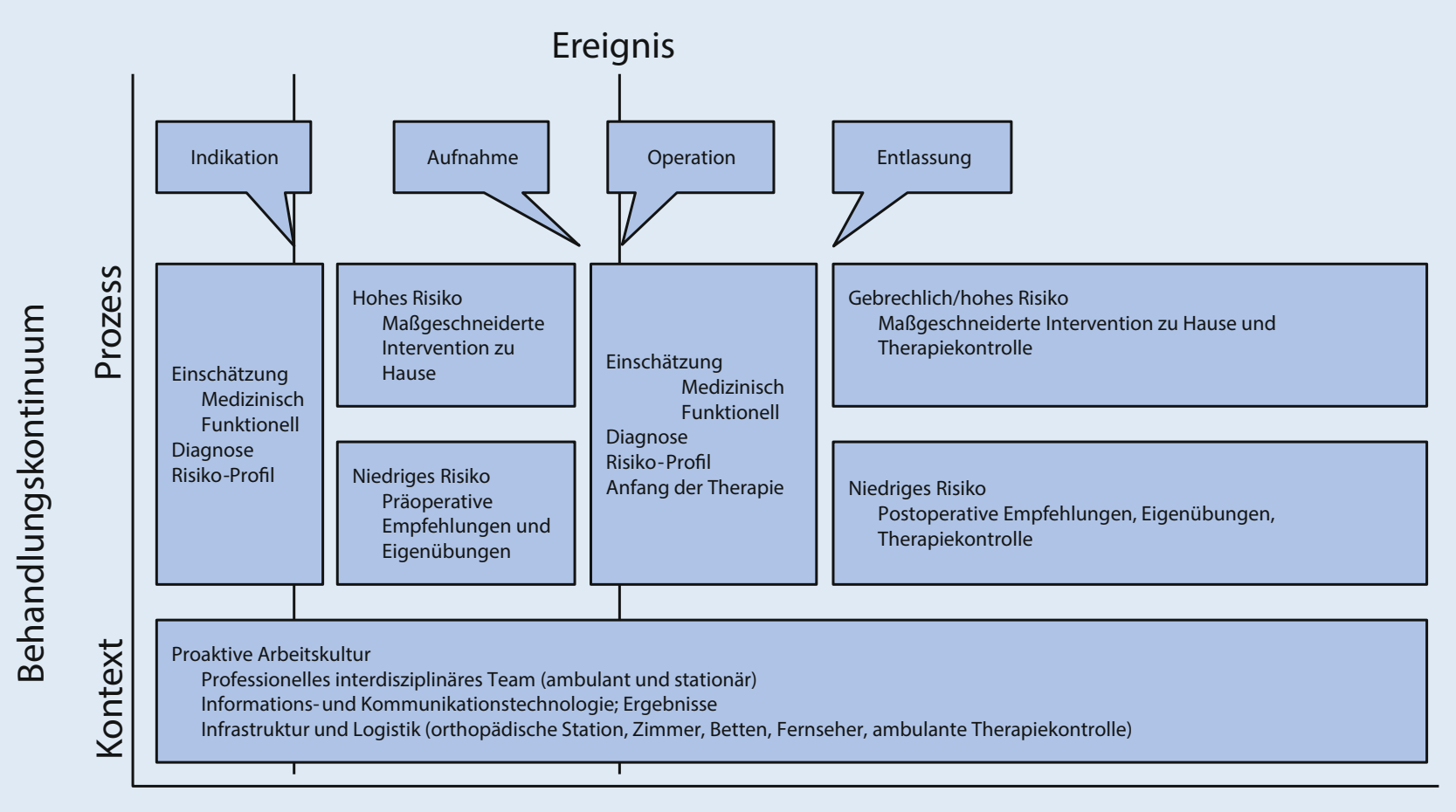

Zeit

Abb. 2 \ Schematische Darstellung der lückenlosen perioperativen Versorgung. Der Prozess beginnt mit einer Einschätzung und Berechnung des Risikoprofils. Elektivpatienten werden in Hoch- und Niedrigrisikogruppen eingruppiert und das bestpassende Rehabilitationsprogramm wird zugeteilt. Der multidisziplinäre Kontext (Team, Technik und Infrastruktur) liefert während der gesamten Prähabilitationsperiode eine proaktive Arbeitskultur [1]

und Mortalität bei größeren abdominellen und kardiochirurgischen Eingriffe identifiziert [20]. Diese Faktoren sind Indikatoren für das Anpassungsvermögen des Patienten (Homöostase und angemessene Antworten auf allostatische Belastungen) bei der Bewältigung psychophysiologischer Einflüsse durch größere Operationen [10]. Zum Beispiel sind Patienten, bei denen größere Abdominaleingriffe geplant sind, mit einer niedrigen kardiopulmonalen Leistungsfähigkeit, beispielsweise einer ventilatorischen Schwelle $(\mathrm{VT})<11 \mathrm{~mL} / \mathrm{kg} / \mathrm{min}$ oder einer maximalen Sauerstoffaufnahme $\left(\mathrm{VO}_{2}\right)<18 \mathrm{~mL} / \mathrm{kg} / \mathrm{min}$, grundsätzlich als Hochrisikopatienten einzustufen [20]. In der Praxis kann diese Leistungsfähigkeit auch mit einfacheren Tests, wie z. B. Steep-ramp-Test und Gehtests (6Minuten-Gehtest [6MWT]), ohne den Gasaustausch gemessen werden [21, 22]. Außerdem können auch andere Tests, wie der Stair-rise-time- und Handgrip-strength-Test, zur Vorhersage der postoperativen Mortalität und beim Entlassungsmanagement verwendet werden $[23,24]$.

\section{Patientenschulung}

Die große Bedeutung der perioperativen Leistungsfähigkeit und des perioperativen Leistungsniveaus wird den Patienten in einer Schulung nahe gelegt, um eine adäquate Wiedergewinnung des präoperativen Leistungsniveaus $\mathrm{zu}$ erreichen. Hierbei erklärt der schulende Physiotherapeut u. a. die ungünstigen Auswirkungen der Bettruhe auf die funktionelle Leistungsfähigkeit, die weitestgehend vermieden werden sollte [25], sowie die Bedeutung einer schnellen Mobilisierung [26] und der körperlichen Aktivität [4, 7]. Zudem wird Hochrisikopatienten mit niedrigem Aktivitätsniveau empfohlen, an der präoperativen Trainingstherapie teilzunehmen, damit sie den negativen Einflüssen der Operation, den anästhesiologischen Prozeduren und den noch sehr restriktiven und patienteninaktivie- renden Pflegestandards besser gewachsen sind.

\section{Trainingstherapie für Hochrisiko- patienten}

Damit Hochrisikopatienten körperlich so aktiv wie möglich bleiben, bekommen sie ein ambulantes Trainingsprogramm in ihrer eigenen häuslichen Umgebung. Dies wird von Physiotherapeuten durchgeführt, die speziell von $\mathrm{BiBo}^{\mathrm{m}}$ ausgebildet wurden. Ziel der Prähabilitation ist die Stärkung des pulmonalen, kardiovaskulären und muskulären Systems, sodass die präoperative Reserve erhöht und das körperliche Adaptationsvermögen auf perioperative Stressoren gesteigert wird [6].

\section{Postoperativ}

\section{Mobilisation und funktionelle Übungstherapie}

Im postoperativen Verlauf sollten die Krankenhausstruktur und die Arbeits- 
weise der unterstützenden Berufe (wie Pflegepersonal und Physiotherapeuten) so organisiert sein, dass der Patient ermutigt wird, aktiv an seiner Genesung teilzunehmen. Der heutige Stand konzentriert sich allerdings eher auf die Versorgung des Patienten im Krankenbett. Dieses System nötigt den Patienten im Bett zu bleiben, auch wenn es keine medizinische Notwendigkeit hierfür gibt [27].

Es ist sehr wichtig, eine gemeinsame Behandlungskultur zu entwickeln, die eine frühzeitige Mobilisation und Aktivierung des Patienten vorsieht.

In der Fast-track-Surgery werden Patienten in einer Umgebung therapiert, in der alle Patienten bereits frühzeitig mobilisiert werden, Übungen zusammen absolvieren und gemeinsame Freizeitaktivitäten durchführen. Jedem Patienten wird empfohlen, so früh wie möglich aus dem Bett aufzustehen; auch wenn erst wenige Stunden seit der Operation vergangen sind [26]. Die Patienten können dadurch sofort ihre Rehabilitationsfortschritte selbst kontrollieren und haben eine frühzeitige Entlassung in einem agileren Zustand als gemeinsames Ziel. Der heutige physiotherapeutische Standard sollte von einem One-size-fits-allStandard, der die gleiche Physiotherapiedauer und die gleichen Inhalte für jeden Patienten vorsieht, in einen individualisierten Standard umgewandelt werden. Mit individuellen Physiotherapiestandards kann bei gleicher Therapiedauer eine bessere Versorgung des Patienten organisiert werden. So brauchen manche Patienten etwas mehr Betreuung, andere sind hingegen mit einer schnellen Risikoeinweisung bereits zufrieden [7]. Die postoperative Genesung sollte objektiv funktionelle Meilensteine (z. B. Sitzen an der Bettkannte, selbstständiges Aufstehen oder Treppenlaufen) beinhalten, die gleichzeitig als Indikatoren für postoperative Komplikationen, für Fortschritte des Trainings oder als Indikator für die Entlassungsmodalität dienen können. Entlassene Hochrisikopatienten trainieren weiterhin, wie in der präoperativen Phase vorzugsweise mit dem gleichen spezialisierten Therapeuten, zu Hause. Niedrigrisikopatienten bekommen postoperative Übungen und Empfehlungen, mit denen sie ihre Therapiefortschritte überwachen können. Der BiBo ${ }^{\mathrm{m} w}$-Prozess ist zu Ende, wenn alle Therapiemeilensteine erreicht sind und der Patient an allen notwendigen Aktivitäten wieder teilnehmen kann [7].

\section{Fallbeschreibung}

Eine 71-jährige Patientin $(1,59 \mathrm{~m}, 61 \mathrm{~kg}$, BMI $24,1 \mathrm{~kg} / \mathrm{m}^{2}$, Charnley-Score B [28]) stellte sich mit einer Coxarthrose vor. Nach entsprechender Aufklärung über die Vor- und Nachteile der operativen Therapie und der konservativen Behandlungsoptionen durch den Orthopäden, den Anästhesisten und den Physiotherapeuten hat sie sich für eine totale Hüftendoprothese (HTEP) entschieden. Sie hat sich außerdem bereit erklärt, an einem präoperativen Screening bzw. einer präoperativen Einschätzung der körperlichen Funktionsfähigkeit beim Physiotherapeuten des Krankenhauses teilzunehmen [19].

Während dieses Screenings 6 Wochen präoperativ beurteilen der Physiotherapeut und die Patientin gemeinsam die aktuelle funktionelle Leistungsfähigkeit (inklusive die Übungstoleranz), um eine Einschätzung des Risikoprofils der postoperativen funktionellen Genesung zu erhalten.

\section{Funktionelle Leistungsfähigkeit}

Die funktionelle Leistungsfähigkeit der unteren Extremitäten wird u. a. mithilfe des Five-times-sit-to-stand-Tests (FTSTST) eingeschätzt [29]. Dieser Test misst die Zeit (in Sekunden) die benötigt wird, um 5-mal hintereinander aus einem Stuhl aufzustehen, ohne die Hände zum Abstützen zu benutzen. Weiterhin wird die Greifkraft („handgrip strength“ [HGS] in kg) der dominanten Hand gemessen. Mit diesem Test wird mit einem Handdynamometer die allgemeine Muskelkraft des Körpers eingeschätzt [30]. Der Höchstwert von 3 Versuchen wird zur Analyse verwendet. Anschließend wird der DeMorton-Mobility-Index benutzt, um das Mobilitätsniveau und die ADL-Aktivitäten $\mathrm{zu}$ evaluieren [31]. Dann wird zusätzlich mit dem Timedup-and-go-Test (TUG-Test, in Sekun- den) das Mobilitätsniveau des Patienten eingeschätzt. Es wird die Zeit gemessen, die benötigt wird, aus einem Stuhl aufzustehen, $3 \mathrm{~m}$ zu gehen, sich umzudrehen und sich wieder auf den Stuhl zu setzen [32]. Die Werte aus dem TUGTest korrelieren mit der funktionellen Genesung nach einer HTEP [33]. Zum Schluss misst der Ten-meter-walk-Test (10mWT, in $\mathrm{m} / \mathrm{s}$.) die Geschwindigkeit beim Gehen in einem normalen Tempo und die Maximalgeschwindigkeit, ohne zu laufen [34].

\section{Übungstoleranz}

Mit dem 6MWT (in Metern) kann eine Aussage über die Ausdauerfähigkeit gemacht werden [35]. Der Patient muss hierbei in $6 \mathrm{~min}$ so weit wie möglich gehen und kann, wenn gewünscht, sich zum Ausruhen hinsetzen.

\section{Ergebnisse des präoperativen Screenings}

Die Patientin führte den FTSTST in $24,22 \mathrm{~s}$ durch $(192 \%$ vom erwarteten Wert [36]). Die Handgreifkraft betrug $17 \mathrm{~kg}$ ( $74 \%$ vom erwarteten Wert [37]). Sowohl der FTSTST als auch der Handgreiftest implizieren eine reduzierte allgemeine körperliche Muskelkraft (- Tab. 1).

Der DEMMI-Fragenbogen ergab 62 von 100 Punkten. Dieser Wert liegt somit unter dem Gruppendurchschnitt von 85 von100 Punkten [38]. Der TUGTest ergab 14,2 s (154\% vom erwarteten Wert [39]), was auf ein schlechteres Mobilitätsniveau deutet (• Tab. 1).

Während des $10 \mathrm{mWT}$ wurde eine Normalgeschwindigkeit von $0,57 \mathrm{~m} / \mathrm{s}$ und eine Maximalgeschwindigkeit von $0,72 \mathrm{~m} / \mathrm{s}$ verzeichnet. Patienten mit einer Normalgeschwindigkeit über $0,58 \mathrm{~m} / \mathrm{s}$ verfügen über ein ausreichendes Mobilitätsniveau, um sich innerhalb der Wohnung zu bewegen. Die maximale Gehgeschwindigkeit von $0,72 \mathrm{~m} / \mathrm{s}$ könnte aber z.B. zur Überquerung einer Straße an einer Fußgängerampel nicht ausreichen. Die vorgegebene Gehgeschwindigkeit unterscheidet sich von Ampel zu Ampel und ist abhängig davon, ob jemand direkt bei Grün losläuft 


\begin{tabular}{|c|c|c|c|}
\hline & Referenzwert & Präoperativ (\%erw.) & Entlassung (\%erw.) \\
\hline FTSTST $(\mathrm{s})^{\mathrm{a}}$ & 12,6 & $24,22(192 \%)$ & $16,2(129 \%)$ \\
\hline HGS (kg) & 23 & $17(74 \%)$ & $22(96 \%)$ \\
\hline $6 \mathrm{MWT}(\mathrm{m})$ & 452,9 & $231(51 \%)$ & 302 (67 \%) \\
\hline DEMMI & n. z. & 62 & 74 \\
\hline TUG $(s)^{a}$ & 9,2 & 14,2 (154\%) & $10,8(117 \%)$ \\
\hline
\end{tabular}

oder erst später in der Grünphase [40, 41].

Die zurückgelegte Strecke während des 6MWT betrug $231 \mathrm{~m}$, nur $51 \%$ vom erwarteten Wert [42]. Dies deutet auf eine geringe Ausdauerfähigkeit hin.

\section{Präoperative Trainingstherapie}

Basierend auf den Ergebnissen des präoperativen Screenings und des Risikoklassifikationsinstruments nach Elings et al. [19] kamen die Patientin und ihr Physiotherapeut zu dem Schluss, dass die Patientin in die Hochrisikogruppe eingestuft werden sollte (z.B. Alter $\geq$ 70 Jahre, Charnley B und TUG $\geq 12,5 \mathrm{~s}$ ). Die Patientin wurde über die Möglichkeit informiert, an der präoperativen Trainingstherapie teilzunehmen, und willigte ein.

Es wurde ein ambulanter Therapieplan für 6 Wochen erstellt. Die funktionelle Trainingstherapie verlief unter Begleitung eines $\mathrm{BiBo}^{\mathrm{mm}}$-zertifizierten Physiotherapeuten und unter Einbeziehung des Ehemanns in der eigenen Wohnung. Der Inhalt der Therapie wurde maßgeschneidert auf die funktionellen Möglichkeiten, das Potenzial und die Bedürfnisse der Patientin. Die Trainingseinheiten konzentrierten sich auf die Steigerung der funktionellen Leistungsfähigkeit bei den für diese Patientin wichtigen alltäglichen Innen- und Außenaktivitäten.

Zum Beispiel gab die Patientin an, das Bedürfnis zu haben, täglich zu ihrer Schwester gehen zu können (Spaziergang von $10 \mathrm{~min}$ ) und wieder kleine Haushaltsaktivitäten durchführen zu können. Daraufhin bekam sie eine Kombination aus begleiteten (Physiotherapeut) und assistierten (Ehemann) Übungen mit 2-mal wöchentlichen Tests, um ihre Fortschritte zu registrieren. Die Übungen beinhalteten u. a. Spaziergänge innerhalb und außerhalb der Wohnung, Treppensteigen ohne und mit Extrabelastung (z. B. Einkaufstaschen und Wäschekörbe) und sich im körperlichen Anspruch steigernde Haushaltsaktivitäten. Diese Übungen der alltäglichen Aktivitäten haben sich bei der substanziellen Steigerung der funktionellen Leistungsfähigkeit und Übungstoleranz effektiver gezeigt als herkömmliche Krafttrainingseinheiten [8].

Die Patientin bekam außerdem die Empfehlung, mithilfe ihrer Verwandtschaft zwischen den Trainingseinheiten körperlich aktiv zu bleiben. Diese Empfehlung hat sie zumeist zusammen mit ihrem Ehemann umgesetzt.

Die funktionelle Leistungsfähigkeit und Übungstoleranz wurde in den 6 Wochen der Prähabilitation ohne zusätzlich auftretende Symptome gesteigert (FTSTST: 16,2 s, $-63 \%$; HGS: $22 \mathrm{~kg},+22 \%$; 6MWT: 302 Metern, $+16 \%$; DEMMI: 74 ; TUG: $10,8 \mathrm{~s},-37 \%$; - Tab. 1).

\section{Stationärer Verlauf}

Die Krankenhausverweildauer betrug insgesamt 3 Tage. Im Vergleich mit anderen Hochrisikopatienten in der Krankenhausdatenbank ist dies eine Reduktion um 1,5-2 Tage.

Die Infrastruktur und Arbeitskultur der orthopädischen Station wurde bereits so geändert, dass ein proaktiver Umgang mit den Patienten eingesetzt hat. Patienten werden vom Behandlungsteam immer wieder motiviert und animiert, mental, sozial und körperlich aktiv zu bleiben. Durch die Einführung eines Gemeinschaftsraums werden die mentalen, so- zialen und körperlichen Aktivitäten stimuliert (gemeinsames Frühstück, Mittagessen, Abendessen und Spaziergänge).

Mit der Physiotherapie wird bereits $4 \mathrm{~h}$ nach der Operation begonnen. Hierdurch wird die funktionelle Unabhängigkeit so schnell wie möglich wiederhergestellt. Mithilfe der Modified-Iowa-Levelof-Assistence-Skala (MILAS; [19]) kann eine Entlassungsentscheidung beschleunigt werden. Mit MILAS werden 5 ADL geprüft, ob sie sicher und mit wieviel Hilfe durchgeführt werden können: aus sitzender Position hinlegen, aus liegender Position hinsetzen, Aufstehen aus sitzender Position, Gehen und Treppensteigen. Jede Aktivität wurde täglich mit 7 Kategorien bewertet: 0 (selbstständig), 1 (unter Supervision), 2 (ein Kontaktpunkt), 3 (2 Kontaktpunkte), 4 (3 oder mehr Kontaktpunkte), 5 (nicht durchführbar trotz maximaler Hilfe) und 6 (nicht durchgeführt aus medizinischen oder sicherheitsrelevanten Gründen). Funktionelle Unabhängigkeit wird bei 0 Punkten des MILAS erreicht.

Die Entwicklung des MILAS ist in - Tab. 2 verzeichnet. Anfänglich $(4 \mathrm{~h}$ nach der Operation) erreichte die Patientin einen Score von 20 Punkten $(3 / 3 / 4 / 4 / 6=20)$. Bereits am 2 . postoperativen Tag erreichte sie 3 Punkte $(1 / 1 / 0 / 0 / 1=3)$ und vorausschauend auf einen 0-Punkte-Score am nächsten Tag bereitete sie sich zusammen mit dem Behandlungsteam auf eine Entlassung am 3. postoperativen Tag vor. Am 3. postoperativen Tag erreichte sie - wie vorhergesagt - die funktionelle Unabhängigkeit (MILAS 0) und wurde nach Hause entlassen. Im Entlassungsbrief wurden die Details der prä- und postoperativen Leistungsfähigkeit und Übungstoleranz eingefügt. Der Patientin wurde empfohlen, sich beim gleichen Physiotherapeuten wie vor der Operation vorzustellen, um die postoperative Trainingstherapie fortzusetzen. Das Ziel sollte sein, zusammen mit ihrem Ehemann das präoperative Leistungsniveau wieder zu erreichen.

Zusammenfassend stufte das präoperative Screening die Patientin in die Hochrisikogruppe für postoperative Komplikationen und einen komplizierten Verlauf ein. Sie wurde darüber 
Tab. 2 Entwicklung des Modified-lowa-Level-of-Assistence-Scores (MILAS) während des stationären Aufenthalts

\begin{tabular}{lllllll} 
& $\begin{array}{l}\text { Hinlegen } \\
\text { (aus Sitzen) }\end{array}$ & $\begin{array}{l}\text { Hinsetzen } \\
\text { (aus Liegen) }\end{array}$ & $\begin{array}{l}\text { Auf- } \\
\text { stehen }\end{array}$ & Gehen & $\begin{array}{l}\text { Treppen- } \\
\text { steigen }\end{array}$ & Total \\
\hline 4 h postoperativ & 3 & 3 & 4 & 4 & 6 & 20 \\
\hline 1. РОT & 3 & 3 & 3 & 1 & 6 & 16 \\
\hline 2. РОT & 1 & 1 & 0 & 0 & 1 & 3 \\
3. РОT & 0 & 0 & 0 & 0 & 0 & 0
\end{tabular}

POT postoperativer Tag; Score: 0 selbstständig; 1 unter Supervision; 2 ein Kontaktpunkt; 3 zwei Kontaktpunkte; 4 drei oder mehr Kontaktpunkte; 5 nicht durchführbar trotz maximaler Hilfe; 6 nicht durchgeführt aus medizinischen oder sicherheitsrelevanten Gründen

informiert, dass sie mit diesem Risikoprofil normalerweise mindestens 5 Tage postoperativ benötigen würde, um funktionell unabhängig zu werden (MILAS = 0 ; [43]), und eine längere Verweildauer in einer stationären Rehabilitation $\mathrm{zu}$ erwarten ist $[19,44]$.

Allerdings war in einer vergangenen Studie an Hochrisikopatienten nachgewiesen worden, dass durch $\mathrm{BiBo}^{\mathrm{Tm}}$ eine Reduktion der Verweildauer von einem Tag erreicht werden könne (von 5,2 Tage auf 4,2 Tage) [44].

Aufgrund dieser Tatsachen beschloss die Patientin, ihre funktionelle Leistungsfähigkeit und Übungstoleranz mithilfe eines spezialisierten Physiotherapeuten und ihres Ehemanns in ihrer eigenen Umgebung aktiv zu steigern. Während der 6 Wochen vor der Operation kontrollierten die Patientin und ihr Physiotherapeut die Fortschritte der Therapie mit Leistungstests. Die Darstellung der Ergebnisse der Leistungstests motivierte die Patientin, sich allmählich von einer Hochrisikopatientin in eine Mediumrisiko- und sogar zu einer Niedrigrisikopatientin zu verbessern. Die Wartezeit auf die Operation wurde somit in eine Trainings- und Aufbauphase umgewandelt.

Wie in $\bullet$ Tab. 1 dargestellt, verbesserte sich die Patientin durch die Aktivitätssteigerung und die kombinierten Trainingstherapien bei allen Tests. Dies führte zu einem besseren Leistungsniveau und einer größeren Übungstoleranz bei der stationären Aufnahme. Auch während der stationären Aufnahme führte sie ihre aktivierenden Übungen fast lückenlos weiter durch. Die MILAS-Scores in • Tab. 2 sind Nachweise für eine schnelle Genesung. Die Patientin und das Behandlungsteam haben sich aufgrund dieses
Verlaufs für eine Entlassung nach Hause statt in eine Reha-Einrichtung entschieden. Im Vergleich zu Verlaufsberichten von anderen, nicht vorbehandelten Hochrisikopatienten ist dies ein sehr schneller Verlauf, ohne dass die Notwendigkeit für eine anschließende stationäre Reha-Behandlung besteht.

\section{Fazit}

Es sollte jedem Patienten bewusst sein, dass ein hohes perioperatives Aktivitätsniveau eine positive Wirkung auf die Genesung und körperliche Kondition nach einem chirurgischen Eingriff hat. Vor allem fragile, gebrechliche Patienten haben ein erhöhtes Risiko auf peri- und postoperative Komplikationen sowie auf eine längere Verweildauer und erreichen erst spät ihr altes Aktivitätsniveau.

Das Better In Better Out ${ }^{\mathrm{Tm}}\left(\mathrm{BiBo}^{\mathrm{rx}}\right)$ Konzept hält es für essentiell, dass $\mathrm{Pa}$ tienten mit einem hohen Risiko eines komplizierten postoperativen Verlaufes mittels Screening der körperlichen Leistungsfähigkeit rechtzeitig identifiziert und informiert werden.

\section{Schlussfolgerung}

Sowohl Hochrisiko- als auch Niedrigrisikopatienten sollten rechtzeitig über die Notwendigkeit eines ausreichenden Aktivitätsniveaus vor, während und nach einem stationären Aufenthalt als schützende Maßnahme vor perioperativen Komplikationen und als Beschleuniger der Genesung bei Elektivoperationen informiert werden. Hochrisikopatienten sollten eine ambulante Trainingstherapie zur Erhöhung der körperlichen Leistungsfähigkeit in ihrer häuslichen Umgebung angeboten werden. Mit einer gestärkten körperlichen Kondition sind diese Patienten den negativen Effekten einer größeren Operation besser gewachsen. Während und auch nach dem stationären Aufenthalt sollten Patienten motiviert und begleitet werden, um ihre objektiv messbaren funktionellen Ziele/Meilensteine zu erreichen. Die eigene Rolle des Patienten im Genesungsprozess ist ein zentraler Punkt. Patienten sollten sowohl fachlich als auch sozial unterstützt und motiviert werden, ihre Trainingsfortschritte selbstständig wahrzunehmen, zu überwachen und weiter auszubauen. Dies soll die eigene Motivation, das Engagement, die Trainingseinhaltung und den Spaß beim Trainieren steigern.

Der zusätzliche Vorteil des $\mathrm{BiBo}^{\mathrm{Tm}}$ Konzepts ist eine Reduktion der allgemeinen Gesundheitskosten, beispielsweise durch die Verkürzung der Verweildauer und durch eine Verringerung der Komplikationen. Außerdem ist eine Abnahme der Indikation für stationäre geriatrische Reha-Maßnahmen zu erwarten.

Heutzutage basiert die Entscheidung zur Operation vor allem auf der Verfügbarkeit eines Operationsteams oder Operationssaals. In der Zukunft sollte aber der Operationszeitpunkt in Abhängigkeit von der klinischen Verfassung des $\mathrm{Pa}$ tienten gewählt werden. Mithilfe eines multidisziplinären Teams wird der $\mathrm{Pa}$ tient entscheiden, ob er sich operieren lässt und ob eine ausreichende körperliche Belastungsfähigkeit besteht.

Änderungen in chirurgischen Techniken haben das Auftreten von vermeidbaren iatrogenen Verzögerungen des Genesungsprozesses, vor allem durch Anpassung im biomedizinischen Bereich reduzieren können. Die Auswirkung dieser Enhanced-recovery-Konzepte auf die Langzeitergebnisse und Verhaltensänderungen (Patient und Team) ist eher eingeschränkt. Die Patientenrolle innerhalb des Gesundheitssystems ändert sich allmählich: Kliniker und Patienten arbeiten immer mehr als Partner am Genesungsprozess zusammen. Damit Genesungsverzögerungen vermieden und die Langzeitergebnisse verbessert werden, sollten medizinische Fachkräfte und Patienten gemeinsam adäquate Behandlungsentscheidungen treffen, 
wobei die Vor- und Nachteile anderer Behandlungsoptionen, die Patientenwünsche, -erwartungen und -umstände in Betracht gezogen werden. Dieses Shared-decision-Making (SDM) wird das Patientenwissen, die Fähigkeit, Behandlungsrisiken richtig einzuschätzen, und die Patient-Behandlungsteam-Kommunikation erhöhen.

In zukünftigen Studien sollte der Mehrwert des SDM bei Patienten mit der Indikation zu großen chirurgischen Eingriffen und auch die Kosteneffektivität von alternativen Behandlungskonzepten weiter untersucht werden.

Aufgrund kultureller Unterschiede sollte die Wirksamkeit und Kosteneffektivität des $\mathrm{BiBo}^{\mathrm{rm}}$-Konzepts und anderer neuer Behandlungskonzepte auch auf internationalem Niveau geprüft werden.

\section{Korrespondenzadresse}

\section{M. Punt}

Institut für Epidemiologie, Maastricht

University, CAPHRI

Postfach 616, 6200 MD Maastricht, Niederlande ilona.punt@maastrichtuniversity.nl

Open access funding provided by Maastricht University.

\section{Einhaltung ethischer Richtlinien}

Interessenkonflikt. I.M. Punt, R. van der Most B.C. Bongers, A. Didden, E.H. J. Hulzebos, J.J. Dronkers und N.L.U. van Meeteren geben an, dass kein Interessenkonflikt besteht.

Dieser Beitrag beinhaltet keine von den Autoren durchgeführten Studien an Menschen oder Tieren.

Open Access Dieser Artikel wird unter der Creative Commons Namensnennung 4.0 International Lizenz (http://creativecommons.org/licenses/by/4.0/deed. de) veröffentlicht, welche die Nutzung, Vervielfältigung, Bearbeitung, Verbreitung und Wiedergabe in jeglichem Medium und Format erlaubt, sofern Sie den/die ursprünglichen Autor(en) und die Quelle ordnungsgemäßnennen, einen Linkzur Creative Commons Lizenz beifügen und angeben, ob Änderungen vorgenommen wurden

\section{Literatur}

1. Hulzebos EH, van Meeteren NL (2016) Making the elderly fit for surgery. Br JSurg 103(2):e12-e15

2. Covinsky KE, Palmer RM, Fortinsky RH, Counsell SR, Stewart AL, Kresevic D, Burant CJ, Landefeld CS (2003) Loss of independence in activities of daily living in older adults hospitalized with medical illnesses: Increased vulnerability with age. J Am Geriatr Soc 51(4):451-458

3. Covinsky KE, Pierluissi E, Johnston CB (2011) Hospitalization-associated disability: "She was probably able to ambulate, but I'm not sure". JAMA 306(16):1782-1793

4. Sourdet S, Lafont C, Rolland Y, Nourhashemi F, Andrieu S, Vellas B (2015) Preventable iatrogenic disability in elderly patients during hospitalization. J Am Med Dir Assoc 16(8):674-681

5. Carli F, Scheede-Bergdahl C (2015) Prehabilitation to enhance perioperative care. Anesthesiol Clin 33(1):17-33

6. Mina SD, Clarke $H$, Ritvo $P$, Leung $Y W$, Matthew AG, Katz J, Trachtenberg J, Alibhai SM (2014) Effect of total-body prehabilitation on postoperative outcomes: A systematic review and meta-analysis. Physiotherapy 100(3):196-207

7. Bongers BC, Dronkers JJ, Hulzebos HJ, Hoogeboom TJ, Buhre WF, van Meeteren NLU (2016) Optimizing perioperative physical therapy care in major elective surgery to improve surgical outcome in high-risk patients: The Better in, Better out ${ }^{\mathrm{TM}}$ concept. Ned Tijdschr Anesthesiol 29(6):134-139

8. de Vreede PL, Samson MM, van Meeteren NL, Duursma SA, Verhaar HJ (2005) Functionaltask exercise versus resistance strength exercise to improve daily function in older women: a randomized, controlled trial. J Am Geriatr Soc 53(1):2-10

9. de Vreede PL, Samson MM, van Meeteren NL, van der Bom JG, Duursma SA, Verhaar HJ (2004) Functional tasks exercise versus resistance exercise to improve daily function in older women A feasibility study. Arch Phys Med Rehabil 85(12):1952-1961

10. Dronkers J, Witteman B, van Meeteren N (2016) Surgery and functional mobility: Doing the right thing at the right time. Tech Coloproctol 20(6):339-341

11. Lipsitz LA, Goldberger AL (1992) Loss of 'complexity' and aging. Potential applications of fractals and chaos theory to senescence. JAMA 267(13):1806-1809

12. Manor B, Lipsitz LA (2013) Physiologic complexity and aging: Implications for physical function and rehabilitation. Prog Neuropsychopharmacol Biol Psychiatry 45:287-293

13. Weston M, Weston KL, Prentis JM, Snowden CP (2016) High-intensity interval training (HIT) for effective and time-efficient pre-surgical exercise interventions. Perioper Med (Lond) 5:2

14. Hoogeboom TJ, Dronkers JJ, van den Ende CH, Oosting E, van Meeteren NL (2010) Preoperative therapeutic exercise in frail elderly scheduled for total hip replacement: a randomized pilot trial. Clin Rehabil 24(10):901-910

15. Oosting E, Jans MP, Dronkers JJ, Naber RH, Dronkers-Landman CM, Appelman-de Vries SM, van Meeteren NL (2012) Preoperative homebased physical therapy versus usual care to improve functional health of frail older adults scheduled for elective total hip arthroplasty: A pilot randomized controlled trial. Arch Phys Med Rehabil 93(4):610-616

16. Ashworth NL, Chad KE, Harrison EL, Reeder BA, Marshall SC (2005) Home versus center based physical activity programs in older adults. Cochrane Database Syst Rev 1:CD004017

17. Glasziou P, Irwig L, Mant D (2005) Monitoring in chronic disease: $A$ rational approach. BMJ 330(7492):644-648

18. Dronkers JJ, Lamberts $H$, Reutelingsperger IM, Naber RH, Dronkers-Landman CM, Veldman A, van
Meeteren NL (2010) Preoperative therapeutic programme for elderly patients scheduled for elective abdominal oncological surgery: A randomized controlled pilot study. Clin Rehabil 24(7):614-622

19. Elings J, van der Sluis $G$, Goldbohm RA, Galindo Garre F, de Gast A, Hoogeboom T, van Meeteren NL (2016) Development of a risk stratification model for delayed inpatient recovery of physical activities in patients undergoing total hip replacement. JOrthop Sports Phys Ther 46(3):135-143

20. Moran J, Wilson F, Guinan E, McCormick P, Hussey J, Moriarty J (2016) Role of cardiopulmonary exercise testing as a risk-assessment method in patients undergoing intra-abdominal surgery: Asystematic review. Br JAnaesth 116(2):177-191

21. Bongers BC, Takken T (2014) The paediatric version of the steep ramp test. J Physiother 60(2):113

22. Awdeh H, Kassak K, Sfeir P, Hatoum H, Bitar H, Husari A (2015) The SF-36 and 6-minute walk test are significant predictors of complications after major surgery. World J Surg 39(6):1406-1412

23. Dronkers JJ, Chorus AM, van Meeteren NL, Hopman-Rock M (2013) The association of preoperative physical fitness and physical activity with outcome after scheduled major abdominal surgery. Anaesthesia 68(1):67-73

24. Reddy $S$, Contreras CM, Singletary B, Bradford TM, Waldrop MG, Mims AH, Smedley WA, Swords JA, Wang TN, Heslin MJ (2016) Timed stair climbing is the single strongest predictor of perioperative complications in patients undergoing abdominal surgery. J Am Coll Surgeons 222(4):559-566

25. Hoogeboom TJ, Dronkers JJ, Hulzebos EH, van Meeteren NL (2014) Merits of exercise therapy before and after major surgery. Curr Opin Anaesthesiol 27(2):161-166

26. Kehlet H (2013) Fast-trackhip and knee arthroplasty. Lancet 381(9878):1600-1602

27. Brown CJ, Redden DT, Flood KL, Allman RM (2009) The underrecognized epidemic of low mobility during hospitalization of older adults. J Am Geriatr Soc57(9):1660-1665

28. Charnley J (1972) The long-term results of low friction arthroplasty of the hip performed as a primary intervention. J Bone Joint Surg $\mathrm{Br}$ 54(1):61-76

29. Suzuki T, Bean JF, Fielding RA (2001) Muscle power of the ankle flexors predicts functional performance in community-dwelling older women. J Am Geriatr Soc 49(9):1161-1167

30. Abizanda P, Navarro JL, Garcia-Tomas MI, LopezJimenez E, Martinez-Sanchez E, Paterna G (2012) Validity and usefulness of hand-held dynamometry for measuring muscle strength in community-dwelling older persons. Arch Gerontol Geriatr 54(1):21-27

31. Jans MP, Slootweg VC, Boot CR, de Morton NA, van derSluis G, van Meeteren NL (2011) Reproducibility and validity of the Dutch translation of the de Morton Mobility Index (DEMMI) used by physiotherapists in older patients with knee or hip osteoarthritis. Arch Phys Med Rehabil 92(11):1892-1899

32. Podsiadlo D, Richardson S (1991) The timed "Up \& Go": A test of basic functional mobi lity for frail elderly persons. J Am Geriatr Soc 39(2 ):142-148

33. Nankaku M, Tsuboyama T, Akiyama H, Kakinoki R, Fujita Y, Nishimura J, Yoshioka Y, Kawai H, Matsuda S (2013) Preoperative prediction of ambulatory status at 6 months after total hip arthroplasty. Phys Ther 93(1):88-93 


\section{Leitthema}

34. Peters DM, Fritz SL, Krotish DE (2013) Assessing the reliability and validity of a shorter walk test compared with the 10-meter walk test for measurements of gait speed in healthy, older adults. J Geriatr Phys Ther 36(1):24-30

35. Kennedy DM, Stratford PW, Wessel J, Gollish JD, Penney D (2005) Assessing stability and change of four performance measures: A longitudinal study evaluating outcome following total hip and knee arthroplasty. Bmc Musculoskelet Disord 6:3

36. Bohannon RW (2006) Reference values for the fiverepetition sit-to-stand test: A descriptive metaanalysis of data from elders. Percept Mot Skills 103(1):215-222

37. Webb AR, Newman LA, Taylor M, Keogh JB (1989) Hand grip dynamometry as a predictor of postoperative complications reappraisal using age standardized grip strengths. J Parenter Enter Nutr 13(1):30-33

38. Macri EM, Lewis JA, Khan KM, Ashe MC, de Morton NA (2012) The de morton mobility index: Normative data for a clinically useful mobility instrument. J Aging Res 2012:353252

39. Bohannon RW (2006) Reference values for the timed up and go test: A descriptive meta-analysis. JGeriatr Phys Ther 29(2):64-68

40. Hoxie RH, Rubenstein LZ (1993) Are older pedestrians allowed enough time to safely cross intersections. Clin Res 41(1):A59-A59

41. Robinett CS, Vondran MA (1988) Functional ambulation velocity and distance requirements in rural and urban communities - a clinical report. Phys Ther 68(9):1371-1373

42. Enright PL, Sherrill DL (1998) Reference equations for the six-minute walk in healthy adults. Am J Respir Crit Care Med 158(5 Pt 1):1384-1387

43. Brunenberg DE, van Steyn MJ, Sluimer JC, Bekebrede LL, Bulstra SK, Joore MA (2005) Joint recovery programme versus usual care: an economic evaluation of a clinical pathway for joint replacement surgery. Med Care 43(10):1018-1026

44. van der Sluis G, Goldbohm RA, Bimmel R, Garre FG, Elings J, Hoogeboom TJ, van Meeteren NLU (2015) What augmented physical activity and empowerment can bring to patients receiving total knee replacement: Content, implementation, and comparative effectiveness of a new functiontailored care pathway in a routine care setting. Biomed Res Int. doi:10.1155/2015/745864 